\title{
The effects of a congested fixture period on physical performance, technical activity and injury rate during matches in a professional soccer team
}

\author{
Alexandre Dellal, ${ }^{1,2}$ Carlos Lago-Peñas, ${ }^{3}$ Ezequiel Rey, ${ }^{3}$ Karim Chamari, ${ }^{2,4}$ \\ Emmanuel Orhant ${ }^{5}$
}

- Additional material is published online only. To view please visit the journal online (http://dx.doi.org/10.1136/ bjsports-2012-091290)

${ }^{1}$ FIFA Medical Excellence Centre, Santy Orthopedicae clinical, sport science and research department, Lyon, France

${ }^{2}$ Tunisian Research Laboratory 'Sport Performance

Optimisation', National Centre of Medicine and Science in Sport (CNMSS), El Menzah, Tunisia

${ }^{3}$ Faculty of Sports Sciences, University of Vigo, Pontevedra, Spain

${ }^{4}$ ISSEP Ksar-Saïd, Manouba University, Manouba, Tunisia ${ }^{5}$ Olympique Lyonnais FC (soccer)

\section{Correspondence to} Alexandre Dellal, OGC Nice (Soccer), Fitness coach department, 177 route de Grenoble, 06201 Nice Cedex 3, France; alexandredellal@gmail.com

Received 21 April 2012 Revised 16 January 2013 Accepted 19 January 2013 Published Online First 19 February 2013

\section{CrossMark}

To cite: Dellal A, LagoPeñas C, Rey E, et al. Br J Sports Med 2015:49: 390-394.

\author{
ABSTRACT \\ Background In modern professional soccer, the ability \\ to recover from official match-play and intense training is \\ often considered a determining factor in subsequent \\ performance.
}

Objective To investigate the influence of playing multiple games with a short recovery time between matches on physical activity, technical performance and injury rates.

Methods The variation of physical (overall distance, light-intensity, low-intensity, moderate-intensity and high-intensity running) and technical performance (successful passes, balls lost, number of touches per possession and duels won) of 16 international players was examined during three different congested periods of matches (six games in 18 days) from the French League and Cup $(n=12)$, and the UEFA Champions' League $(n=6)$ during the 2011-2012 season and compared with that reported in matches outside these periods. Data were collected using a computerised match analysis system (Amisco). Injury rate, time loss injuries, as well as the mechanism, circumstances and severity of the injury were also analysed.

Results No differences were found across the six successive games in the congested period, and between no congested and the three congested periods for all the physical and technical activities. The total incidence of injury (matches and training) across the prolonged congested periods did not differ significantly to that reported in the non-congested periods. However, the injury rate during match-play was significantly higher during the congested period compared with the noncongested period $(p<0.001)$. The injury rate during training time was significantly lower during the congested period compared with the non-congested periods ( $p<0.001)$. The mean lay-off duration for injuries was shorter during the congested periods compared with the non-congested periods $(9.5 \pm 8.8$ days vs $17.5 \pm 29.6$ days, respectively $p=0.012$, effect sizes $=0.5$ ). Conclusions Although physical activity, technical performance and injury incidence were unaffected during a prolonged period of fixture congestion, injury rates during training and match-play and the lay-off duration were different to that reported in matches outside this period.

\section{INTRODUCTION}

In modern professional soccer, the ability to recover from official match play and intense training is often considered to be a determining factor in subsequent performance. ${ }^{1}$ Professional soccer players are often required to play competition matches with only 2-3 days' recovery in between. In such conditions, the maintenance or improvement of the player's activity is determined not only by appropriate conditioning but also by the ability of the body systems to recover and regenerate after multiple stress stimuli. ${ }^{2-5}$

Several investigations have analysed the physical activity profiles and injury rates of professional soccer players during intense periods of matches. ${ }^{6-12}$ Unexpectedly, those studies did not reveal any differences in the distances covered at various speeds and in the injury risk across successive matches played in a short time period. However, except for the work of Carling $e \mathrm{al}^{7}$ in these previous studies, physical performance and injury rates were examined across only two or three consecutive games played over a timescale of 3-7 days.

The effects of a single prolonged period of fixture congestion (eight matches in 26 days) on a top-level team were investigated. ${ }^{7}$ The major findings revealed that the high-intensity running (HIR) and the injury rate of the players remained unaffected. However, these findings are not conclusive due to specific limitations: (1) the number of players studied varied from match to match; (2) only six players took part in every game as starters or substitutes; (3) only one outfield player completed every game studied and the goalkeeper was included in that study. Further research is necessary to explore the effects of short recovery times between matches on physical activity and injury rates. Additionally, there is a lack of data regarding the effects of prolonged periods of fixture congestion on technical performance.

Therefore, two hypotheses were proposed in the current study: (1) physical and technical performances would differ across games over a single congested period of 3 weeks (six games), and depending on the soccer season time periods, (2) match injury rates and severity would be greater during the prolonged period of fixture congestion compared with the habitual one-game per week period. The aim of this work was twofold. First, to investigate the influence of playing multiple games with a short recovery time between matches on physical activity, technical performance and injury rates during three different congested periods of matches throughout the season. Second, to compare previous performance profiles to those reported in matches outside these congested fixture periods. 


\section{METHODS}

\section{Study design}

To analyse the effect of games repetition on physical performance and injury rates, we have examined the time-motion characteristics and injuries of the highest level of the French soccer league (ie, 'Ligue 1') during both training and official games. All the matches were played during three different congested fixture periods of the 2011-2012 season (first period from 6 to 24 August 2011; second period from 10 to 27 September 2011 and the third period from 15 October 2011 to 2 November 2011). Each congested fixture period consisted of six consecutive matches separated by 3 days. Congested period corresponded to a period when the team played two games in a week, and consequently, a non-congested period was a period with one match in a week. Official matches included 11 matches in the French 'Ligue 1', one match in the French League Cup and six matches in the UEFA Champions' League, starting at either 17:00, 19:00 or 20:45 Data from players completing at least $75 \mathrm{~min}$ in every game of each congested fixture period were included in the analysis. The methodologies and procedures applied in the current study closely follow those used by Carling et al. ${ }^{7}$

The postmatch recovery strategies used are those traditionally used in soccer and which have been previously validated for professional soccer players. The day after each match, all players were advised to use static stretching (involving three bilateral sets of $30 \mathrm{~s}$ held stretches to the hamstring, quadriceps, gastrocnemius and adductor muscles), to receive a whole-body manual massage (three players never solicited massage) and to immerse their lower body in an alternating cold and hot bath as previously recommended. ${ }^{13}{ }^{14}$ For diet monitoring, each player was given a meal plan (food and hydration) composed in collaboration with the club's nutritionist for both immediately after the match and the days after the match.

\section{Participants}

The players included in the study met two basic criteria: (1) completing at least $75 \mathrm{~min}$ in every game (six matches) of each congested period, and (2) because the physical loading of goalkeepers differs from that of field players, they were not included in the study. Altogether, 16 outfield players (goalkeepers were excluded) (first period $n=5$, second period $n=6$ and third period $n=5)$ from a squad of 22 professional players participated in the present study (age: $24.3 \pm 3.2$ year; height: $178.1 \pm 4.2 \mathrm{~cm}$; body mass: $76.9 \pm 4.3 \mathrm{~kg}$ ). Only four players participated in every game in all the three studied congested fixture periods. The study conforms to the code of ethics of the World Medical Association and the standards for research's recommendation of the Helsinki Declaration. ${ }^{15}$ The protocol was approved by the local university ethics committee and the involved club, and all subjects gave written informed consent prior to enrolling in the study. To ensure team and player confidentiality, all performance and injury data were anonymised before analysis.

\section{Match performance}

Player movements were tracked with a computerised player tracking system (AMISCO Pro, Sport-Universal Process, Nice, France) at a sampling rate of $25.0 \mathrm{~Hz}$ providing approximately 2.5 million data points per match. ${ }^{16-18}$ This system examines simultaneously the time-motion characteristics of all players differentiating when they have the collective ball possession or not. Randers $e a^{18}$ have recently evaluated the reliability and validity of AMISCO Pro for quantifying displacement velocities during match-related activities relative to data obtained using timing gates. An intraobserver and interobserver reliability analysis was conducted using five elite soccer players. Two trained observers tracked each player on two occasions (weeks 1 and 3) and the coefficient of variation (CV) was determined to assess reliability. The intraobsrever and interobserver $\mathrm{CV}$ for total distance, walking, running, high-speed running, HIR and very HIR was $<2 \%$ with the exception of sprinting, which was $<3 \%$.

\section{Physical performance}

The total distance covered during all training and official matches was measured. Four thresholds were used to evaluate the distances run in four categories of running intensity: $0.0-12.0 \mathrm{~km} / \mathrm{h}$ (walking and light-intensity running, Light-IR); $12.1-18.0 \mathrm{~km} / \mathrm{h}$ (low-intensity running, Low-IR); $18.1-21.0 \mathrm{~km} / \mathrm{h}$ (moderateintensity running, MIR) and $>21.0 \mathrm{~km} / \mathrm{h}$ (HIR). The absolute data were corrected to a minute-by-minute analysis of the distance covered in each category (distance divided by effective playing time) in order to enable equal comparison of performance between soccer players. ${ }^{19}$

\section{Technical performance}

The technical performance indicators include the percentage of successful passes, number of balls lost, total number of touches per possession and percentage of duels won. Bradley et $a l^{20}$ observed good interobserver and intraobserver reliability for the number and type of recorded technical events $(k>0.9)$.

\section{Injury definition, classification, severity, injury rate and injury collection procedures}

Injury data referred to time loss injuries, which occurred when a player was incapable of taking full part in future soccer training or matches owing to physical complaints. ${ }^{21}$ The same club doctor diagnosed all injuries, and an injured player was considered to be injured until the club doctor cleared him for participation in full training or matches. The team physician prospectively recorded the mechanism (traumatic or overuse), circumstances (match or training) and severity of the injury. Injuries were classified into four categories of severity, according to the length of absence from full training sessions and matches, including the day of injury: slight (1-3 days), minor (4-7 days), moderate ( $8-28$ days) and major (more than 28 days). ${ }^{19}$ Match exposure was defined as play between teams from different clubs. Training exposure corresponded to team-based and individual physical activities under the control or guidance of the team's coaching or fitness staff that aimed at maintaining or improving players' soccer skills or physical condition. ${ }^{9}$ Injury rate was calculated as all injuries per $1000 \mathrm{~h}$ of soccer (training + matches), and injuries per $1000 \mathrm{~h}$ of training and injuries per $1000 \mathrm{~h}$ of matches separately. The methodologies and definitions of injury used in the present study closely follow those recommended by the Injury Consensus Group of FIFA Medical Assessment and Research Center ${ }^{21}$ and are similar to those used in other investigations of professional soccer players. ${ }^{79}$

\section{Statistical analyses}

All statistical analyses were conducted using the statistical package SPSS for Macintosh (V.18.0, Chicago, Illinois, USA). Results are expressed as means $\pm \mathrm{SD}$, and the statistical analyses are according to the model of Carling $\mathrm{et} \mathrm{al.}^{7}$ The normality of the data of the distribution was tested by the Kolmogorov-Smirnov test with the Lilliefors' correction. Changes of physical activity (overall distance covered and that in Light-IR, Low-IR, MIR and HIR) and skill- 
Table 1 Changes in measures of physical performance in professional soccer players over three prolonged congested fixture periods of six successive matches ( $n=16$ outfield players)

\begin{tabular}{|c|c|c|c|c|c|c|c|}
\hline Distances covered ( $\mathrm{m} / \mathrm{min}$ of the effective playing time) & Match 1 & Match 2 & Match 3 & Match 4 & Match 5 & Match 6 & Statistical difference \\
\hline Overall distance & $200.5 \pm 23.7$ & $209.7 \pm 22.7$ & $214.8 \pm 24.5$ & $211.8 \pm 19.6$ & $194.5 \pm 11.3$ & $184.5 \pm 13.2$ & $p=0.072$ \\
\hline High-intensity running, HIR & $9.5 \pm 4.0$ & $10.2 \pm 4.9$ & $11.0 \pm 4.7$ & $11.7 \pm 3.4$ & $8.5 \pm 2.7$ & $8.4 \pm 5.0$ & $p=0.622$ \\
\hline Moderate-intensity running, MIR & $9.9 \pm 3.6$ & $10.4 \pm 3.3$ & $11.9 \pm 2.8$ & $10.9 \pm 2.1$ & $9.1 \pm 2.7$ & $8.6 \pm 3.5$ & $p=0.277$ \\
\hline Low-intensity running, Low-IR & $43.9 \pm 12.9$ & $46.3 \pm 12.6$ & $48.2 \pm 14.5$ & $42.6 \pm 5.3$ & $38.3 \pm 9.9$ & $38.8 \pm 9.3$ & $p=0.467$ \\
\hline Light-intensity running Light-IR & $133.8 \pm 23.8$ & $142.7 \pm 16.4$ & $143.6 \pm 15.3$ & $146.5 \pm 22.0$ & $138.5 \pm 15.1$ & $128.5 \pm 6.9$ & $p=0.368$ \\
\hline
\end{tabular}

The values are the mean distances covered in every match of the three congested periods.

related performance (the percentage of successful passes, number of balls lost, total number of touches per possession and percentage of duels won) were evaluated using a one-way analysis of variance (ANOVA) with repeated measures. The one-way ANOVA was used to evaluate differences across: (1) six consecutive games over an 18-day-period, and (2) games played during three different congested fixture periods in the same season. In the event of a difference occurring, Tukey's post hoc tests were used to identify any localised effects. Fisher's exact test was used to compare injury rates (number of injuries per $1000 \mathrm{~h}$ of exposure) for matches during and outside the congested fixture period. The Mann-Whitney test was used to compare severity during and outside the congested period. Statistical significance was set at $\mathrm{p}<0.05$. The $95 \%$ CIs and intersubject CV were also calculated. Cohen's d effect sizes (ES) for identified statistical differences were determined. When calculating ES, pooled SD were applied due to the absence of a control group. ES with values of $0.2,0.5$ and 0.8 were considered to represent small, medium and large differences, respectively. ${ }^{22}$

\section{RESULTS}

Across the six successive matches analysed per player during the three congested periods, no differences were reported for the overall distance covered or that covered in HIR, MIR, Low-IR or Light-IR by the 16 outfield players analysed (table 1). The respective mean CVs across the successive games for the previous five categories of movement intensities were: $9.6 \%$ (CI $9.4 \%$ to $9.8 \%$ ), $41.1 \%$ (CI $37.7 \%$ to $45.4 \%$ ), $31.1 \%$ (CI $29.2 \%$ to $33.3 \%$ ), $27.6 \%$ (CI $25.9 \%$ to $29.5 \%$ ) and $11.1 \%$ (CI $10.8 \%$ to $11.4 \%)$. An analysis of the technical actions showed that the number of duels won, the number of ball contacts per possession, the percentage of successful passes and the number of balls lost did not differ significantly across the six successive matches during the three congested periods (table 2).

A comparison of physical performance in matches played across the three separate periods (August, September, October and November) revealed significant increases in the overall distance covered. More total distance was covered in games played in October-November (Period 3) compared with August
(Period 1) $\quad(215.2 \pm 23.0$ vs $192.9 \pm 11.1 \mathrm{~m} / \mathrm{min}$, respectively $\mathrm{p}<0.001$, ES for these differences ranged from 0.6 to 1.2 ; figure 1). However, no differences were found between the periods for the distance covered in HIR $(8.9 \pm 3.1$ vs $9.8 \pm 4.4$ vs 10.7 $\pm 4.7 \mathrm{~m} / \mathrm{min}, \mathrm{p}=0.308)$, MIR $(9.3 \pm 3.0$ vs $10.1 \pm 3.8$ vs 11.3 $\pm 2.8 \mathrm{~m} / \mathrm{min}, \mathrm{p}=0.066)$, Low-IR $(42.2 \pm 10.1$ vs $43.9 \pm 12.9$ vs $46.4 \pm 13.8 \mathrm{~m} / \mathrm{min}, \mathrm{p}=0.456)$ and Light-IR $(132.5 \pm 8.8$ vs 138.6 \pm 10.2 vs $143.4 \pm 28.4 \mathrm{~m} / \mathrm{min}, \mathrm{p}=0.122$ ). The total incidence of injury (matches and training) across the prolonged congested periods did not differ significantly to that reported in noncongested periods. However, injury rate during match-play was significantly higher during the congested period compared with the non-congested period $(p<0.001)$. On the other hand, injury rate during training time was significantly lower during the congested period compared with the non-congested periods $(p<0.001)$. The mean lay-off duration for injuries was shorter during the congested periods compared with the non-congested periods $(9.5 \pm 8.8$ days vs $17.5 \pm 29.6$ days, respectively $\mathrm{p}=0.012$, $\mathrm{ES}=0.5)$. The majority of injuries were classified as moderate (39.8\%), followed by slight (28.1\%), minor (27.9\%) and major (4.2\%) during the congested period, but no difference was observed with the non-congested periods (table 3 ). Injury rates, injury circumstances (match vs training), severity and mechanism of injury according to the grouping (congested periods vs noncongested periods) were presented in table 3 .

\section{DISCUSSION}

The present study is the first to investigate the influence of playing multiple games with a short recovery time between matches on physical activity, technical performance and injury rates in a professional soccer team. Three different congested periods of matches (six games in 21 days) during the 2011-2012 season were examined. The major findings were that physical activities and technical performances were unaffected during the congested fixture periods. Although the total incidence of injury during the congested periods of fixture was similar to that reported in matches outside these periods, injury rates during training and matches play and the mean lay-off duration varied during the congested periods.

Table 2 Changes in measures of skill-related performance in professional soccer players over three prolonged congested fixture periods of six successive matches ( $n=16$ outfield players)

\begin{tabular}{|c|c|c|c|c|c|c|c|}
\hline Technical performance & Match 1 & Match 2 & Match 3 & Match 4 & Match 5 & Match 6 & Statistical difference \\
\hline Duels won (\%) & $52.9 \pm 16.3$ & $51.7 \pm 16.5$ & $54.8 \pm 16.4$ & $59.6 \pm 9.5$ & $44.3 \pm 16.5$ & $56.7 \pm 7.8$ & $p=0.588$ \\
\hline Mean number of ball contact per possession & $2.2 \pm 0.3$ & $2.1 \pm 0.3$ & $2.2 \pm 0.4$ & $2.1 \pm 0.3$ & $1.9 \pm 0.2$ & $2.2 \pm 0.1$ & $p=0.695$ \\
\hline Number of balls lost & $12.8 \pm 4.2$ & $13.4 \pm 5.6$ & $10.8 \pm 3.6$ & $12.6 \pm 5.1$ & $11.2 \pm 3.6$ & $12.7 \pm 2.6$ & $p=0.580$ \\
\hline Successful passes (\%) & $84.9 \pm 6.1$ & $84.7 \pm 7.6$ & $83.2 \pm 7.7$ & $84.4 \pm 5.1$ & $85.3 \pm 8.6$ & $87.7 \pm 3.8$ & $p=0.900$ \\
\hline
\end{tabular}

The values are the mean distances covered in every match of the three congested periods. 


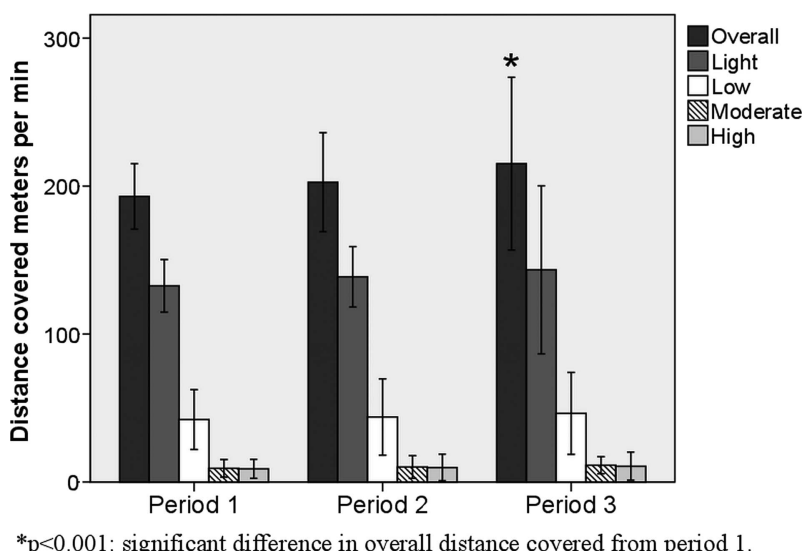

Figure 1 Comparison of distance covered at different running intensities across three separate periods of a congested calendar during the same season. The values are the mean distances covered over the six matches of the three congested periods. ${ }^{*} p<0.001$ : significant difference in overall distance covered from period 1.

The lack of statistical difference in the distances covered at different intensities across the six successive matches for the team as a whole is generally in line with previous findings showing that physical performances are not affected by a short time-frame between successive matches. ${ }^{6-12}$ However, except for the work of Carling et $a l^{7}$ these previous studies investigated sporadic congested fixture schedules and only analysed physical performance across two or three consecutive matches within a short time-frame. The findings from the current study, together with those of Carling et al, ${ }^{7}$ suggest that during a certain period professional soccer players can cope with a prolonged period of fixture congestion. A larger sample size than the one used in the present study, combined with the knowledge of the contextual factors that can potentially affect performance, such as the match location, match status, quality of the opponent and environmental conditions, might be necessary to enable better detection of real systematic variations in the distance covered by players during periods of fixture congestion.

Table 3 Injury rates, injury circumstances, severity and mechanism of injury classified according to congested period versus non-congested period

\begin{tabular}{|c|c|c|}
\hline & Congested period & Non-congested period \\
\hline \multicolumn{3}{|c|}{ Injury rate per $1000 \mathrm{~h}$ exposure } \\
\hline Overall & $14.4(13.7-14.9)$ & $15.6(15.1-16.3)$ \\
\hline Match & $43.3^{*}(33.3-57.5)$ & $18.6(16.3-21.3)$ \\
\hline Training & $4.6^{*}(3.2-5.8)$ & $14.6(12.2-17.1)$ \\
\hline \multicolumn{3}{|c|}{ Injury circumstances \% } \\
\hline Match & 75.8 & 29.6 \\
\hline Training & 24.2 & 70.4 \\
\hline \multicolumn{3}{|c|}{ Injury severity, \% } \\
\hline Slight & 28.1 & 48.2 \\
\hline Minor & 27.9 & 14.8 \\
\hline Moderate & 39.8 & 22.2 \\
\hline Major & 4.2 & 14.8 \\
\hline \multicolumn{3}{|c|}{ Mechanism of injury, \% } \\
\hline Traumatic & 44.2 & 44.4 \\
\hline Overuse & 55.8 & 55.6 \\
\hline
\end{tabular}

The present study also revealed that the technical performance did not vary across the six consecutive games. These results suggest that this period of successive matches did not induce a high level of fatigue on players. These results are similar to those provided by previous studies. ${ }^{23-26}$ A further limitation is that only individual technical indicators were analysed. It would appear that these indicators could not reflect the collective behaviour of the team. Future studies should include collective technical indicators such as ball possession.

Fatigue in soccer was commonly analysed according to a short congested period of matches, ${ }^{6} 7{ }^{9-12}$ but no studies have demonstrated if the physical and technical activities decreased or varied according to the stages of the season. In this context, the present study showed that the overall distance covered was greater in the third period (October-November), whereas no differences were observed in the other speed thresholds. Moreover, given that only matches from the first half of the season were examined, variations in physiological and physical responses to match-play should be taken into account at different stages of the season.

The results of the present study are in contrast with those of Carling $e t ~ a l^{7}$ who indicated that there was no difference in injury rates between congested and non-congested matchperiods. Although the present results show that injury rates and severity are similar (combining training and matches) during the prolonged period of fixture congestion compared with outside this period, differences were observed when training and matches were separated. This decrease in the training injury rate during congested fixtures could be explained by the fact that technical staff used low-intensity exercise training and that the players controlled their activity in order to be able to repeat high performance during the close consecutive matches in the congested periods. The higher injury incidence in competition during the congested periods of the fixture compared with that reported outside these periods could be explained by the increased match exposure. However, the injury rate in the present study was lower than those found by previous studies with, for example, a rate of 50.3 versus 43.3 injury/ $1000 \mathrm{~h}$ of match exposure, in comparison with Carling et al This could be due to the player rotation strategy used in the present study's investigated team, which is dependent on the number of elite players composing the squad and the coach' strategy. ${ }^{4} 7$ In line with the findings of Carling et $a l^{7}$ the number of lay-off days was much lower during the non-congested periods, despite the fact that the present study results revealed a higher number of lay-off days than those found by Carling et $a l^{7}$ (9.5 vs 2.0, respectively). This lay-off difference could be related to the injury severity and to the different strategies used by the medical staff in each case. In the present study, we found that $55 \%$ of injuries were caused by overuse, which is much lower than the $76 \%$ found by Dupont et $a l^{9}$ It is probable that these results may suggest that the recovery strategies used in the present study's studied club between matches led to neuromuscular recovery and reduced the risk of injury associated with overuse.

Additional research using a larger sample of clubs and across different countries is necessary as the present cohort included players from only one club. The patterns observed might therefore only be a reflection of this particular team. Another limitation is that the players analysed were not exactly the same across the three different congested periods of fixture congestion. Several players participated just in one congested period. The considerable number of competitive matches and the risk of injury and fatigue result in rotations of players in the starting 
line-up throughout the season. Future studies should analyse how performance varies in the same group of players during different periods of the season and from season to season. Moreover, an analysis of the potential variations in physiological responses to match-play across a similar prolonged period of fixture congestion is also warranted. Data on objective markers of fatigue such as plasma creatine kinase concentration, combined with subjective measures of performance, could provide additional information on the individual and team response to fixture congestion. Finally, future studies should determine how long high-level performance can be maintained playing multiple games with a short recovery time between matches and to avoid an increased rate of injury.

\section{CONCLUSION}

The present study is the first that has examined the variation of key factors of soccer performance (physical, technical and injury rate) during three consecutive congested fixture periods compared with non-congested competitive match periods in a professional soccer team playing the UEFA Champion's League. Although physical activity, technical performance and injury incidence were unaffected during a prolonged period of fixture congestion, the injury rates during training and match-play and the lay-off duration were different to those reported in matches outside this period.

\section{What are the new findings?}

- The present study is the first to explore the pattern of physical activity and injury rates of playing multiple games with a short in-between soccer matches recovery time over three consecutive prolonged periods of fixture congestion during the same season (each congested fixture period consisted of six consecutive matches).

- This is the only study that analyses the effects of a prolonged period of fixture congestion on technical activity, which is one of the main soccer performance determinants.

- A congested soccer calendar may increase injury risk during matches in professional soccer players.

- Physical activities and technical performances are unaffected during congested fixture periods.

\section{How might it impact on clinical practice in the near}

future?

- This study shows how technical and medical staff could manage a congested soccer calendar in order to limit injury risk during consecutive matches.

- The coach, physiologist and medical staff will pay special attention to the recovery strategies when players participate in matches during congested fixture periods.

Correction notice This article has been corrected since it was published Online First. The correspondence address was originally incorrect, as well as the affiliation addresses.

Contributors All authors have (1) provided substantial contributions to conception and design, acquisition of data and/or analysis and interpretation of data; (2) drafted the article and/or revising it critically for important intellectual content and (3) given the final approval of the version to be published.

Competing interests None.
Ethics approval This study meets the standards for research with the recommendation of the Helsinki Declaration. However, the usual appropriate ethics committee clearance required was not sought as the present data arose as a condition of employment in which player performance was routinely measured over the course of the competitive season.

Provenance and peer review Not commissioned; externally peer reviewed.

\section{REFERENCES}

1 Mohr M, Krustrup P, Bangsbo J. Fatigue in soccer: a brief review. J Sports Sci 2005;23:593-9.

2 Kraemer WJ, French DN, Paxton NJ, et al. Changes in exercise performance and hormonal concentrations over a big ten soccer season in starters and non-starters. J Strength Cond Res 2004;18:121-8.

3 Ekstrand J, Hägglund $M$, Waldén $M$. Injury incidence and injury patterns in professional football: the UE FA injury study. Br J Sports Med 2011;45:553-8.

4 Ekstrand J, Walden M, Hägglund M. A congested football calendar and the wellbeing of players: correlation between match exposure of European footballers before the World Cup 2002 and their injuries and performances during that World Cup. Br J Sports Med 2004;38:493-7.

5 Hägglund $M$, Walden M, Bahr R, et al. Methods for epidemiological study of injuries to professional football players: developing the UEFA model. Exposure and injury in Swedish elite football: a comparison between seasons 1982 and 2001. Br J Sports Med 2005;39:340-6.

6 Carling C, Dupont G. Are declines in physical performance associated with a reduction in skill-related performance during professional soccer match-play? J Sports Sci 2011;29:63-71.

7 Carling C, Le Gall F, Dupont G. Are physical performance and injury risk in a professional soccer team in match-play affected over a prolonged period of fixture congestion? Int J Sports Med 2012;33:36-42.

8 Carling C, Orhant E, Le Gall F. Match injuries in professional soccer: inter-seasonal variation and effects of competition type, match congestion and positional role. Int J Sports Med 2010;31:271-6.

9 Dupont G, Nedelec M, McCall A, et al. Effect of 2 soccer matches in a week on physical performance and injury rate. Am J Sports Med 2010;38:1752-8.

10 Lago-Peñas C, Rey E, Lago-Ballesteros J, et al. The influence of a congested calendar on physical performance in elite soccer. J Strength Cond Res 2011;25:2111-17.

11 Odetoyinbo K, Wooster B, Lane A. The effect of a succession of matches on the activity profiles of professional soccer players. In: Reilly T, Korkusuz F, eds. Science and football VI. UK, Routledge, 2007:105-10.

12 Rey E, Lago-Peñas C, Lago-Ballesteros J, et al. The effects of a congested fixture period on the activity of elite soccer players. Biol Sport 2010;27:181-5.

13 Ascensão $A$, Leite $M$, Rebelo $A$, et al. Effects of cold water immersion on the recovery of physical performance and muscle damage following a one-off soccer match. J Sports Sci 2011;29:217-25.

14 Shirrefs SM, Sawka MN, Stone M. Water and electrolyte needs for football training and match-play. J Sports Sci 2006;24:699-707.

15 Winter EM, Maughan RJ. Requirements for ethics approvals. J Sports Sci 2009;27:985.

16 Carling C, Bloomfield J, Nelsen L, et al. The role of motion analysis in elite soccer: contemporary performance measurements techniques and work-rate data. Sports Med 2008;38:839-62.

17 Dellal A, Chamari C, Wong DP, et al. Comparison of physical and technical performance in European professional soccer match-play: the FA Premier League and La LIGA. Eur J Sport Sci 2011;11:51-9.

18 Randers MB, Mujika I, Hewitt A, et al. Application of four different football match analysis systems: a comparative study. J Sports Sci 2010;28:171-82.

19 Castellano J, Blanco-Villaseñor A, Alvarez D, et al. Contextual variables and time-motion analysis in soccer. Int J Sports Med 2011;32:415-21.

20 Bradley PS, O'Donoghue P, Wooster B, et al. The reliability of Prozone MatchViewer: a video-based technical performance analysis system. Int J Perform Anal Sport 2007:7:117-29.

21 Fuller CW, Ekstrand J, Junge A, et al. Consensus statement on injury definitions and data collection procedures in studies of football (soccer) injuries. Scand J Med Sci Sports 2006;16:83-92

22 Cohen J. Statistical power analysis for the behavioural sciences. 2nd edn. Hillsdale, NJ: Lawrence Erlbaum, 1988.

23 Dellal A. Analyze of the soccer player physical activity and of its consequences in the training: special reference to the high intensities intermittent exercises and the small sided-games (Doctoral thesis). Strasbourg, France, University of Sport Sciences, 2008.

24 Rampinini E, Impellizzeri FM, Castagna C, et al. Technical performance during soccer matches of the Italian Serie A league: effect of fatigue and competitive level. J Sci Med Sport 2009;12:227-33.

25 Krustrup P, Mohr M, Steensberg A, et al. Muscle and blood metabolites during a socce game: implications for sprint performance. Med Sci Sports Exerc 2006;38:1165-74.

26 Lago-Penas C, Dellal A. Ball possession strategies in elite soccer according to the evolution of the match-score: the influence of situational variables. J Hum Kin 2010;25:93-100 